\title{
Kidney-Pulmonary Crosstalk from Pathophysiological Perspective
}

\author{
Sharifa Audi Salsabila ${ }^{1}$ (D), Alfian Nur Rosyid ${ }^{2}$ (D), Maulana Antiyan Empitu ${ }^{3}$ (D), \\ Ika Nindya Kadariswantiningsih ${ }^{4}$, Satriyo Dwi Suryantoro $^{5}$ (D), Mutiara Rizki Haryati ${ }^{\text {(D) }}$, \\ Mochammad Thaha ${ }^{5^{*}}$, Yid Yukuke Suzuki $^{6}$ (i) \\ ${ }^{1}$ Faculty of Medicine, Universitas Airlangga, Surabaya, Indonesia. \\ ${ }^{2}$ Department of Pulmonology and Respiratory Medicine, Faculty of Medicine, Universitas Airlangga/Universitas Airlangga Hospital, \\ Surabaya, Indonesia. \\ ${ }^{3}$ Department of Anatomy, Histology, and Pharmacology, Faculty of Medicine, Universitas Airlangga, Surabaya, Indonesia. \\ ${ }^{4}$ Department of Medical Microbiology, Faculty of Medicine, Universitas Airlangga, Surabaya, Indonesia. \\ ${ }^{5}$ Department of Internal Medicine, Faculty of Medicine, Universitas Airlangga/Universitas Airlangga Hospital, Surabaya, Indonesia. \\ ${ }^{6}$ Department of Nephrology, Juntendo University, Tokyo, Japan.
}

\begin{tabular}{l} 
ARTICLE INFO \\
\hline Article history: \\
Received 24 November 2021 \\
Received in revised form 13 January \\
2022 \\
Accepted 24 January 2022 \\
Available online 30 January 2022 \\
\hline Keywords: \\
Acute kidney injury, \\
Acute respiratory distress syndrome, \\
Chronic kidney disease, \\
Kidneys, \\
Lungs. \\
\hline
\end{tabular}

\begin{abstract}
Lungs and kidneys are distant organs which are functionally related in physiological and pathological contexts. Acute lung injury (ALI) or acute respiratory distress syndrome (ARDS) are common complications in acute kidney injury (AKI) or acuteon-chronic kidney disease. On the other hand, there is a considerable risk of AKI in respiratory diseases such as ARDS and chronic obstructive pulmonary disease (COPD). From a pathophysiological point of view, the kidney-lung crosstalk involves interdependency in the regulation of fluid hemodynamic, acid-base and electrolyte balance, and carbon dioxide partial pressure. Aside from the closely related function, the crosstalk may also occur by non-classical mechanisms such as through activation of systemic inflammation, excessive cytokine release, and the formation of autoantibody which targets both kidneys and lungs. This review discussed several disease mechanisms by which kidney and lungs affect each other or are simultaneously affected by pathological processes. Particularly, this review discussed some specific mechanisms in lungs and kidneys, such as how hypoxemia and hypercapnia induced by ARDS may reduce kidney function and how distance injury on kidney may affect the development of non-cardiogenic edema lungs.
\end{abstract}

\section{INTRODUCTION}

Lungs and kidney are two distant organs with seemingly unrelated primary functions. Nevertheless, in many ways, the two organs are closely related both in physiological and pathological contexts. In physiological settings, both kidney and lungs engage in a collaborative function keeping the balance of acid-base, maintaining the harmony of carbon dioxide partial pressure, regulating bicarbonate concentration, controlling the blood pressure, and save-guarding the fluid homeostasis. $^{1}$

In a disease context, the last two years of coronavirus disease 2019 (COVID-19) pandemic has brought a real example of kidney-lung crosstalk into a spotlight. As a native respiratory disease, the primary organ affected by COVID-19 are the airways and lungs. However, kidney involvement is one of the most frequent complications found in hospitalized patients with COVID-19. Although most of COVID-19 mortality is due to hypoxemia, there is also considerable mortality caused by other organ failures. A study of 5,449 adult individuals with COVID-19 who were admitted to hospitals across metropolitan New York, reported that $36 \%$ of the study subjects developed acute kidney injury (AKI). ${ }^{2}$ The study found that AKI was primarily seen amongst patients with respiratory failure, particularly those who required mechanical ventilation. ${ }^{3}$ Studies in China and Europe reported that the rate of AKI in

*Corresponding author: mochthaha@fk.unair.ac.id 
COVID-19 patients ranged from $0.5 \%$ to $27 \%$, depending on the study population. ${ }^{3}$ Regardless of the wide range of $\mathrm{AKI}$ rates, kidney involvement has become possible complication that may hinder the fruitful outcome of therapy in respiratory diseases.

On the other hand, kidney injury may yield consequences to distant organs, including the lungs. Both AKI and end-stage renal diseases (ESRD) may cause acidosis or volume overload which trigger acute pulmonary edema. ${ }^{4}$ Other than the traditional complications, non-traditional complications such as oxidative stress and systemic inflammation may also cause inflammatory lung injury. ${ }^{\frac{5}{}}$ In a condition with a high mortality rate such as AKI, managing complications that can worsen the risk of death is one of the most important aspects of therapy.

Given the previous consideration, the crosstalk between kidney and lungs in the disease context is a topic of interest in this review. This review discussed several mechanisms in which kidney and lungs are specifically affected. From the primary organ point-of-view, this review classified the condition into three categories: systemic conditions which affect both kidney and lungs, primary pulmonary and respiratory diseases which affect kidney, and primary kidney diseases which affect the lungs and respiratory system (Figure 1). In this review, we focused on discussing examples of each category to illustrate how a disease may affect both organs or a disease in kidney can affect the lungs and vice versa.

\section{Systemic conditions which affect both organs}

Several systemic diseases, particularly immunemediated diseases, are found to affect specifically both kidney and lungs. A pulmonary-renal syndrome is a term coined to describe a clinical condition in which intraalveolar hemorrhage and crescentic glomerulonephritis occur simmultanously. ${ }^{6}$ This condition represents a clinical urgency which may lead to an emergency condition given the impending dysfunction of kidney and lungs. This syndrome is suspected in patients with hemoptysis without any clear cause such as pneumonia, bronchiectasis, or carcinoma. Both respiratory symptoms and renal manifestations such as anemia, proteinuria, and hematuria can be found in pneumo-renal syndrome. ${ }^{6}$ This syndrome is prototypically caused by Goodpasture syndrome specifically targeting kidney and lungs, aside from various other diffuse autoimmune diseases such as systemiclupus erythematosus, eosinophilic granulo-

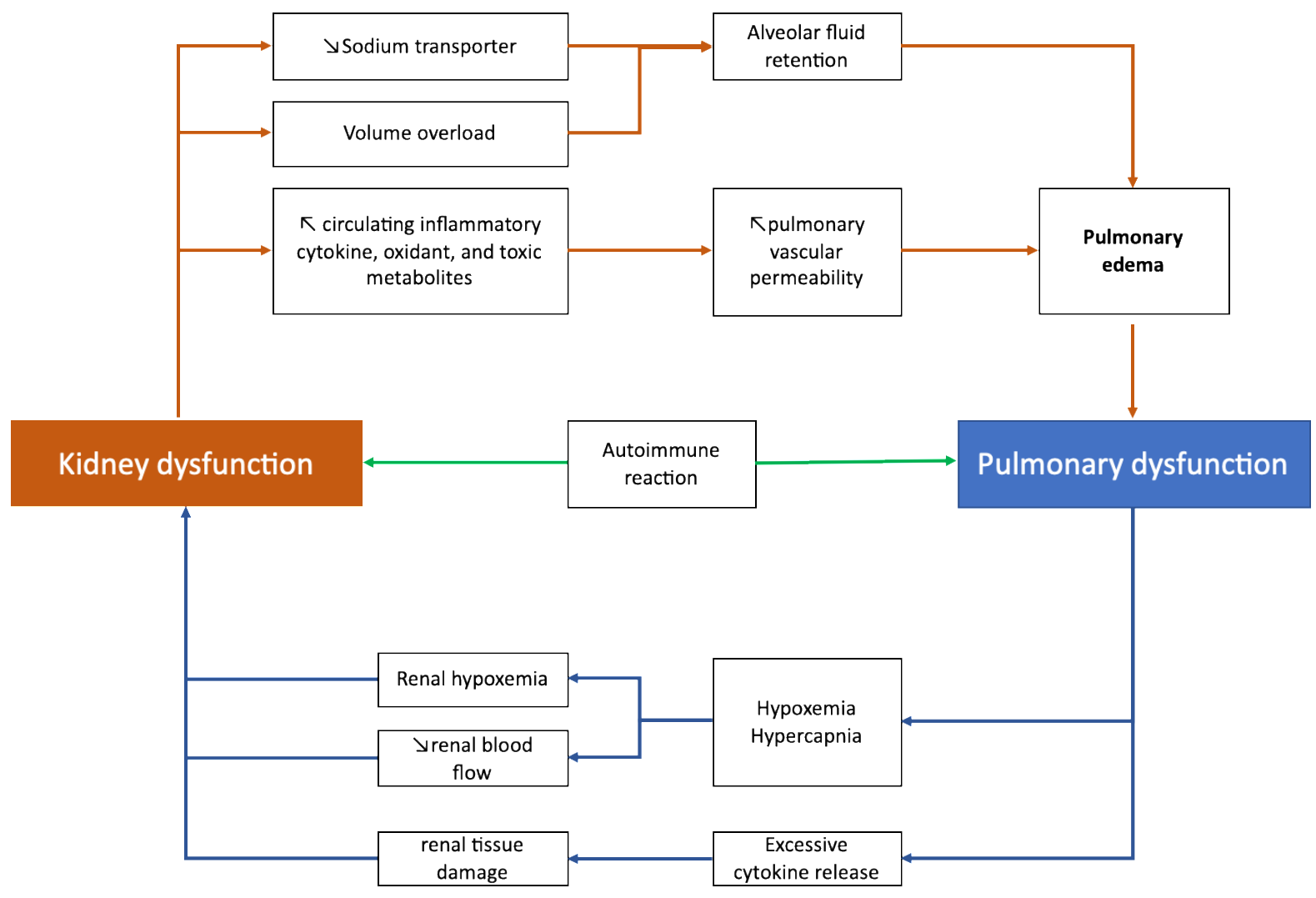

\footnotetext{
$\longrightarrow$ Condition affecting both kidney and lung simmultaneously

Lung condition affecting kidney
}

Figure 1. Pathophysiological sequence describing the kidney-pulmonary crosstalk 
matosis with polyangitis, post-streptococcal glomerulonephritis, rheumatoid arthritis, and systemic sclerosis. ${ }^{7}$ Thus, we discussed the mechanism of how both kidney and lung injury occur in Goodpasture syndrome.

\section{Autoimmunity targeting kidney and lungs}

Goodpasture syndrome is an autoimmune disease specific to kidney and lungs. Epidemiologically, Goodpasture syndrome is considered as a rare disease with an incidence of 1 case per million people per year. Despite its low incidence, $20 \%$ of rapidly progressive glomerulonephritis was caused by anti-glomerular basement membrane. $^{8}$

From a pathophysiological point of view, the structural damage of kidney and lungs may occur concomitantly in Goodpasture syndrome. This syndrome is mediated by anti-glomerular basement membrane (anti-GBM) antibodies. Thus, a serum antibody test with positive anti-GBM indicates the diagnosis of this syndrome. In most patients with Goodpasture syndrome, the antibody targets against $28 \mathrm{kD}$ monomeric subunit of non-collagenous domain of the alpha 3 chain of type IV collagen (alpha3[IV]NC1). ${ }^{9}$

Interestingly, the autoantibody in Goodpasture syndrome specifically targets the basement membrane in the alveoli and glomeruli even though the basement membrane is found everywhere. The clear answer behind this phenomenon is not obvious. However, the epitope accessibility of the alveolar and glomerular basement membrane is one of the possible factors that cause preferential binding of the autoantibody in Goodpasture syndrome. ${ }^{6}$ The conformational structure of alpha3[IV]NC1 in the alveoli and glomeruli makes it readily available for circulating antibodies. There is also a genetic factor such as individuals with certain types of human leukocyte antigen (HLA) which increase susceptibility to Goodpasture syndrome. ${ }^{10}$

Theoretically, the underlying mechanism which target basement membrane in both alveoli and glomeruli would generate clinical manifestations in kidney and lungs. However, about $10 \%$ of individuals with Goodpasture syndrome have isolated lung pathology without kidney involvement. ${ }^{-}$The syndrome may initially manifest as non-specific symptoms such as weakness and fatigue. The condition then progresses gradually or rapidly into AKI accompanied by pulmonary edema and hemorrhage. ${ }^{11}$

In kidney biopsy, the kidney lesion in Goodpasture syndrome appears as crescentic glomerulonephritis. ${ }^{12}$ The kidney immunofluorescence staining of patients with Goodpasture syndrome shows linear staining for $\mathrm{IgG}$ on the GBM. The chest X-ray usually shows bilateral patchy parenchymal consolidations. ${ }^{6}$ There is no clear treatment for this disease given its rarity. However, since it is an immunemediated disease, the available treatments are immunosuppressive agents.

\section{Autoimmunity non-specific to kidney and lungs}

Anti-neutrophil cytoplasmic antibody (ANCA)associated vasculitides (AAV) is the most common cause of pulmonary-renal syndrome, responsible for $20 \%$ of the case. ${ }^{13}$ ANCA-AAV is a group of small vasculitides with circulating ANCA and pauci immune, meaning there is few or negative immunofluorescence deposit. ${ }^{14}$ ANCAAAV targets a diffuse range of organs such as the heart, kidney, lungs, skin, and nervous system.

In ANCA-AAV, the autoantibodies commonly target proteinase-3 (PR3) and myeloperoxidase (MPO) which bind to ANCA forming PR3-ANCA [cytoplasmic ANCA (C-ANCA)] or MPO-ANCA [perinuclear ANCA (P-ANCA)]. ${ }^{15}$ PR3 and MPO are abundantly present in the cytoplasm of neutrophils and monocytes. Both the presence of cytoplasmic and perinuclear ANCA complex may trigger neutrophil degranulation. The neutrophil degranulation prompts oxidative stress release and endothelial lysis causing small vessel vasculitis. ${ }^{16}$

\section{Pulmonary conditions which affect kidney}

The mechanism of renal involvement in respiratory diseases can be a direct or indirect mechanisms, which in many cases are bidirectional. This review discussed how kidney injury can be induced in acute respiratory distress syndrome (ARDS) and systemic inflammatory response syndrome (SIRS) as an example of both direct and indirect mechanisms of kidney involvement.

\section{Hypoxemia and hypercapnia}

Many experimental studies show that acute injury which primarily occurs in the lungs may cause kidney injury. ${ }^{17}$ A study in 634 patients with ARDS showed that $68 \%$ of patients developed AKI. ${ }^{17}$ ARDS is a condition characterized by hypoxemia and bilateral lung infiltrates. It is a result of the unresolved inflammatory response to local insult in the respiratory system or systemic conditions. The finding of increased risk of AKI incidence among hospitalized individuals with ARDS indicated that the kidney-lung crosstalk is relevant in the context of ARDS. ${ }^{17}$

There are several factors in ARDS which may directly affect kidney function. Hypoxemia during ARDS may reduce renal blood flow in a dependent manner to the blood oxygen concentration. ${ }^{18}$ Kidney is an organ with high oxygen consumption, which makes it vulnerable to hypoxic injury. ${ }^{19}$ The reduced renal blood flow triggers a tubulo-glomerular feedback loop which 
subsequently increases kidney vascular resistance and blunted vasodilatory response. ${ }^{19}$ This may explain the fluid resistance which usually occurs in individuals with a chronic respiratory disorder. ${ }^{20}$ Another consequence of reduced ventilation is hypercapnia, which may trigger acid-base imbalance. From a physiological standpoint, the lungs are organs in which oxygen and carbon dioxide exchange take place. This process is a critical step in ensuring the oxygen supply and keeping the acid-base balance.

The use of mechanical ventilation could also determine the fate of kidney injury. The hemodynamic state may change during mechanical ventilation which involves increased positive chest pressure accompanied by reduced cardiac output and venous return. These two factors may further reduce renal blood flow and glomerular filtration rate if not managed carefully. ${ }^{20}$ Additionally, mechanical ventilation may also induce neuro-hormonal changes which activate the reninangiotensin-aldosterone system (RAAS) and sympathetic nervous system which may further decrease renal blood flow. ${ }^{20}$

Aside from the acute insult of the lungs, the chronic hypoxemia and hypercapnia resulted from chronic obstructive pulmonary disease (COPD) may also affect kidney. COPD increased the mortality risk among individuals with chronic kidney disease (CKD) with a hazard ratio of 1:22. ${ }^{21}$ A study involving 189,561 individuals with COPD reported that the prevalence of AKI in the cohort was $128 / 100,000$ person-years, which is higher than its prevalence in the general population. ${ }^{21}$ A study involving 22,080 subjects with COPD found that CKD risk among the population with COPD was 1.6 higher than the population without COPD. ${ }^{22}$ The direct mechanism of CKD in COPD is not fully clear. It is suggested that individuals with COPD generally also develop atherosclerosis and chronic hypertension, which may induce kidney injury in the long term. However, there is evidence that individuals with COPD exhibited tissue hypoxia, which affects kidney and other organs such as liver. ${ }^{21}$

\section{Cytokine release syndrome}

The indirect mechanism of kidney involvement in respiratory disease is via systemic inflammatory response. Massive alveolar epithelial damage which occurs in ALI/ARDS can trigger systemic inflammation. The main effectors of systemic inflammation are the inflammatory cytokines: tumor necrosis factor (TNF)- $\alpha$, interleukin (IL)-6, and IL1 $\beta .{ }^{23}$ Various conditions such as unresolved inflammation, sepsis, or hemophagocytic syndrome may trigger excessive cytokines release which cause exacerbated inflammatory responses, termed as 'cytokine storm' or cytokine release syndrome. In several respiratory infections such as COVID-19, the incidence of cytokine release syndrome has been documented. ${ }^{23}$

The exact mechanism by which cytokine storm may induce renal damage is still unclear. The circulating pro-inflammatory cytokines may activate the local renal resident cells triggering tubular and endothelial dysfunction. ${ }^{24}$ Cytokines which seem relevant to kidney damage in cytokine storm are IL-6, which could induce the renal endothelial cells to secrete chemokines that increase vascular permeability and induce vascular leakage, which further decreases renal blood flow and glomerular filtration rate. ${ }^{23}$ The activation of CD4+ T cells in the tubular interstitial also triggers cellular damage, apoptosis, kidney tissue remodeling, and fibrosis. ${ }^{24}$

\section{Kidney diseases which affect the lungs}

It has been long established that CKD and AKI are linked to pulmonary complications. Certain factors in CKD and AKI may increase the risk of pulmonary complications. Several traditional risks include volume overload, electrolyte abnormalities, metabolic acidosis, and uremia. The non-traditional risks of pulmonary complication include oxidative stress and systemic inflammation, which is affected by multiple conditions in CKD such as poor sleep quality, poor control of blood pressure, and microbiota dysbiosis. , $25,26^{-1}$

\section{Volume overload}

The most important risk of pulmonary complication in AKI is volume overload. In AKI, water retention causes increased vascular hydrostatic pressure causing fluid leakage into the interstitial space. In 1950, it was reported that AKI might cause pulmonary injury demonstrated as abnormal chest X-ray. ${ }^{27}$ Regardless of the cause, volume overload potentially triggers cardiogenic pulmonary edema, which may lead to respiratory failure. This phenomenon has also been observed in CKD, which is known as the uremic lung. By introducing dialysis therapy in 1950, many believed that uremic lung would no longer be a problem. However, long after introducing fluid removal through dialysis, the mortality rate remains high, indicating that the problem is more than just cardiogenic pulmonary edema. ${ }^{27}$

\section{Non-cardiogenic pulmonary edema}

Studies in the experimental model showed that interstitial edema occurs regardless of volume overload, pointing mechanisms other than increased vascular hydrostatic pressure are involved. One of the critical factors causing interstitial edema is increased albumin permeability of pulmonary vasculature during AKI. ${ }^{28}$ The shift of albumin to the alveolar space from the capillary space lead to decreased oncotic pressure within 
the capillary. Thus, the fluid moves from pulmonary capillary to the alveolar space.

Additionally, dysregulation of salt transporter in pulmonary vasculature such as epithelial sodium channel (ENaC), Na-K-ATPase, and aquaporin-5 exaggerate the fluid leakage into pulmonary interstitial. ${ }^{28}$ In physiological conditions, ENaC and Na-K-ATPase pump sodium from the alveolar space into the alveolar epithelial cells, which causes water to shift out of the alveolar space. Down-regulation of $\mathrm{ENaC}$ and $\mathrm{Na}-\mathrm{K}$ ATPase pumps in AKI causes impaired sodium transport, leading to fluid retention in the alveolar cavity. ${ }^{28}$

\section{Unresolved inflammation}

As kidney plays a critical role in the clearance of immune mediators, disruption in renal function generates a risk of imbalance in immune homeostasis. Evidence showed that elimination of circulating pro-inflammatory cytokines are dampened in AKI and CKD. ${ }^{5}$ Data from patients with AKI showed a high level of circulating IL$6 .{ }^{24}$ Pro-inflammatory IL-6 can induce direct injury in pulmonary endothelial or recruit resident cells, thus inducing subsequent lung inflammation, neutrophil trapping, and tissue damage. ${ }^{24}$

The decreased clearance of toxic metabolites and circulating pro-oxidant may also change the dynamic of pulmonary vasculatures. The increased level of circulating lipocalin-2, chemokine ligand-2 (CXCL2), can promote pulmonary vascular permeability which leads to edema. The increased pulmonary vascular permeability also cause the inflow of immune-competent cells into the alveolar cavity, which further induces alveolar inflammation and remodeling. ${ }^{24}$

\section{Kidney-Pulmonary Crosstalk in COVID-19}

The kidney-pulmonary crosstalk in COVID-19 is a unique interaction, since this viral infection can manifest in different way and the pathophysiology is not always obvious. Given its uniqueness and the time urgency of this topic, this review decided to discuss this in a separate sections.

There are ample of evidence that AKI occurred in individuals with COVID-19 that previously developed severe respiratory symptom, thus causing deteriorated renal function. In United States and Europe, the prevalence of AKI among critically ill patients with COVID-19 ranged between 20-40\%. ${ }^{29}$ This indirectkidney injury in critically ill patients could be caused by multifactors such as predisposed cardiovascular disease, metabolic disease, and immune response dysregulation caused by prolonged viremia. ${ }^{3}$ Subsequently, SARSCoV-2-related immune response dysregulation may induce cytokine release syndrome discussed in the previous section.
However, there are also some findings which stated that SARS-CoV-2 may also infect kidney capilary endothelial directly. Post mortem data from individuals with SARS-CoV-2 indicated that viral particles were found within renal endothelial cells, tubular epithelium, and podocytes. ${ }^{3}$ This finding prompted a conversation whether SARS-CoV-2 not only has a tissue tropism towards upper respiratory epithelia, but also other endothelial cells and even other types of cells.

Podocytes are pericyte-like cells which are phenotypically different from most endothelial and epithelial cells. ${ }^{30}$ The present of angiotensin converting enzyme 2 (ACE2) in the kidney's tubular cell and podocytes are thought to be one of factors that enable a direct infection of SARS-CoV-2 among these cell types. ${ }^{29}$ Podocytes injury, including one caused by viral infection, may trigger further structural changes in the integrity of slit diaphragm (SD) which is critical in preserving plasma protein and filtering the blood. ${ }^{30}$ The changes in cytoskeletal formation subsequently cause footprocess effacement and disruption of SD. If this pathological process persist in a relatively long time, these event may trigger podocyte detachement from glomerular basement membrane, apoptosis, and glomerulosclerosis. ${ }^{30}$ Together with tubular injury, renal infection by SARS-CoV-2 may cause accute tubular necrosis, glomerulosclerosis, collapsing glomerulopathy, and protein leakage to urinary space. ${ }^{29}$

Given the two possible pathways of kidney injury involving indirect and direct mechanisms, supportive care such as removal of nephrotoxin, tight monitoring of kidney function (serum creatinie, cystatin-C, urine output, and albuminuria), and haemodynamic monitoring become critically important in hospitalized patients with COVID-19 and risk factors of kidney injury. ${ }^{29}$ Adjusting fluid balance to suit the volume responsiveness is also critical to avoid overloads and volume depletion which may burden the injured kidney. Renal replacement theraphy could also be considered in individuals with volume overloads and poor response to conservative treatment. ${ }^{3,29}$

\section{SUMMARY}

From a mechanism standpoint, kidney and lungs interact functionally in both physiological and pathological contexts. The interdependencies between kidney and lungs in a physiological context include the essential function in regulating the acid-base balance, carbon dioxide partial pressure, and fluid homeostasis. There is evidence that dysfunction which initially includes one organ can induce dysfunction in the other organs in a pathological context. For instance, hypoxemia in ARDS may cause AKI by triggering 
neuro-hormonal RAAS feedback, increasing renal vascular resistance, and reducing renal blood flow. On the other hand, volume overload and chronic inflammation in CKD are prone to cause pulmonary edema. This bidirectional crosstalk prompted holistic management of acute and chronic kidney and lung diseases by considering possible complications and outcomes of both organs. In certain condition, such as individuals with COVID-19 and risk of kidney injury, regular monitoring of haemodinamic and kidney function (serum creatinie, cystatin-C, urine output, and albuminuria) may help to detect an alarming indication for further aggressive therapy. In individuals with poor response to supportive treatment, rapid resolution therapy (RRT) can be considered to prevent further decline of kidney function and renal pathologic deterioration.

\section{Acknowledgments}

Thank you to the Director of Universitas Airlangga Hospital, Surabaya Indonesia, Dean of Juntendo University, Tokyo, Japan, Head Division of Nephrology Juntendo Univerisity Tokyo Japan.

\section{Confict of Interest}

The author stated there is no conflict of interest in this study.

\section{Funding}

This study does not receive any funding.

\section{Authors' Contributions}

Conceiving manuscript, analyzing, reviewing and revising: MT. Writing manuscript: SAS. Literature searching: SAS, MAE, IKN, SDS, MRH. Reviewing: YS. Content and gramatical revising: ANR. Provide funding: MT. All authors contributed and have approved the final version.

\section{REFERENCES}

1. Visconti L, Santoro D, Cernaro V, Buemi M, Lacquaniti A. Kidney-Lung Connections in Acute and Chronic Diseases: Current Perspectives. J Nephrol. 2016;29(3):341-8. [CrossRef] [PubMed]

2. Fisher M, Neugarten J, Bellin E, Yunes M, Stahl L, Johns TS, et al. AKI in Hospitalized Patients with and without COVID-19: A Comparison Study. J Am Soc Nephrol. 2020;31(9):2145-57. [CrossRef] [PubMed]

3. Hirsch JS, Ng JH, Ross DW, Sharma P, Shah HH, Barnett RL, et al. Acute Kidney Injury in Patients Hospitalized with COVID-19. Kidney Int. 2020;98(1):209-18. [CrossRef] [PMC free access]

4. Vieira Jr JM, Castro I, Curvello-Neto A, Demarzo S, Caruso P, Pastore Jr L, et al. Effect of Acute
Kidney Injury on Weaning from Mechanical Ventilation in Critically Ill Patients. Crit Care Med. 2007;35(1):184-91. [PubMed]

5. Empitu MA, Kadariswantiningsih IN, Thaha M, Nugroho CW, Putri EAC, El Hakim Z, et al. Determiner of Poor Sleep Quality in Chronic Kidney Disease Patients Links to Elevated Diastolic Blood Pressure, hs-CRP, and Blood-count-based Inflammatory Predictors. Indones Biomed J. 2019;11(1):100-6. [CrossRef]

6. Greco A, Rizzo MI, De Virgilio A, Gallo A, Fusconi M, Pagliuca G, et al. Goodpasture's Syndrome: A Clinical Update. Autoimmun Rev. 2015;14(3):246-53. [PubMed]

7. Gallagher H, Kwan JTC, Jayne DRW. Pulmonary Renal Syndrome: A 4-Year, Single-Center Experience. Am J Kidney Dis. 2002;39(1):42-7. [PubMed]

8. Tang W, McDonald SP, Hawley CM, Badve S V, Boudville NC, Brown FG, et al. Anti-Glomerular Basement Membrane Antibody Disease is an Uncommon Cause of End-Stage Renal Disease. Kidney Int. 2013;83(3):503-10. [PubMed]

9. Cui Z, Zhao M-H. Advances in Human Antiglomerular Basement Membrane Disease. Nat Rev Nephrol. 2011;7(12):697-705. [CrossRef]

10. Yang R, Cui Z, Zhao J, Zhao M-H. The Role of HLA-DRB1 Alleles on Susceptibility of Chinese Patients with Anti-GBM Disease. Clin Immunol. 2009;133(2):245-50. [PubMed]

11. Lahmer T, Heemann U. Anti-Glomerular Basement Membrane Antibody Disease: A Rare Autoimmune Disorder Affecting the Kidney and the Lung. Autoimmun Rev. 2012;12(2):169-73. [PubMed]

12. Pedchenko V, Bondar O, Fogo AB, Vanacore R, Voziyan P, Kitching AR, et al. Molecular Architecture of the Goodpasture Autoantigen in Anti-GBM Nephritis. N Engl J Med. 2010;363(4):343-54. [CrossRef] [PubMed]

13. West SC, Arulkumaran N, Ind PW, Pusey CD. Pulmonary-Renal Syndrome: A Life Threatening but Treatable Condition. Postgrad Med J. 2013;89(1051):274-83. [CrossRef] [PubMed]

14. Rowaiye OO, Kusztal M, Klinger M. The Kidneys and ANCA-Associated Vasculitis: from Pathogenesis to Diagnosis. Clin Kidney J. 2015;8(3):343-50. [PubMed]

15. Furuta S, Jayne DRW. Antineutrophil Cytoplasm Antibody-Associated Vasculitis: Recent Developments. Kidney Int. 2013;84(2):244-9. [PubMed]

16. Kallenberg CGM. Pathogenesis of ANCAAssociated Vasculitides. Ann Rheum Dis. 2011;70(Suppl 1):i59-63. [CrossRef] [PubMed]

17. Panitchote A, Mehkri O, Hastings A, Hanane T, Demirjian S, Torbic H, et al. Factors Associated with Acute Kidney Injury in Acute Respiratory Distress Syndrome. Ann Intensive Care. 2019;9(1):1-10. [PubMed]

18. Sharkey R,Mulloy EM,O’Neill SJ. Acute Effects of Hypoxaemia, Hyperoxaemia And Hypercapnia on 
Renal Blood Flow in Normal and Renal Transplant Subjects. Eur Respir J.1998;12(3):653-7. [CrossRef] [PubMed]

19. Ricksten S-E, Bragadottir G, Redfors B. Renal Oxygenation in Clinical Acute Kidney Injury. Annu Updat Intensive Care Emerg Med 2013. 2013;67995. [CrossRef]

20. Kuiper JW, Groeneveld ABJ, Slutsky AS, Plotz FB. Mechanical Ventilation and Acute Renal Failure. Crit Care Med. 2005;33(6):1408-15. [PubMed]

21. Lai C-C, Wu C-H, Wang Y-H, Wang C-Y, Wu VC, Chen L. The Association between COPD and Outcomes of Patients with Advanced Chronic Kidney Disease. Int J Chron Obstruct Pulmon Dis. 2018;13:2899. [PubMed]

22. Chen C-Y, Liao K-M. Chronic Obstructive Pulmonary Disease is Associated with Risk of Chronic Kidney Disease: A Nationwide CaseCohort Study. Sci Rep [Internet]. 2016;6(1):25855. Available from: https://doi.org/10.1038/srep25855 [CrossRef] [PubMed]

23. Fajgenbaum DC, June CH. Cytokine Storm. N Engl J Med. 2020;383(23):2255-73. [CrossRef] [PubMed]

24. Ahmadian E, Hosseiniyan Khatibi SM, Razi Soofiyani S, Abediazar S, Shoja MM, Ardalan M, et al. COVID - 19 and Kidney Injury:
Pathophysiology and Molecular Mechanisms. Rev Med Virol. 2021;31(3):e2176. [CrossRef] [PubMed]

25. Thaha M, Kadariswantiningsih IN, Empitu MA. Association of High Blood Pressure with Elevated Oxidative Stress, Inflammatory Marker and Albuminuria in Chronic Kidney Disease Patients. J Med. 2019;20:12-8. [CrossRef]

26. Kadariswantiningsih IN, Thaha M, Nugroho CW, Empitu MA. Thiol-Producing Microbiota of the Intestine Modulate Oxidative Stress and Inflammation in Chronic Kidney Disease. Bali Med Journal; 2019 Vol. 8(1): 307-31. [CrossRef]

27. Scheel PJ, Liu M, Rabb H. Uremic Lung: New Insights into a Forgotten Condition. Kidney Int. 2008;74(7):849-51. [PubMed]

28. Guo C, Wu T, Zhu H, Gao L. Aquaporin 4 Blockade Attenuates Acute Lung Injury through Inhibition of Th17 Cell Proliferation in Mice. Inflammation. 2019;42(4):1401-12. [PubMed]

29. Ronco C, Reis T, Husain-Syed F. Management of Acute Kidney Injury in Patients with COVID-19. Lancet Respir Med. 2020;8(7):738-42. [CrossRef]

30. Empitu MA, Kadariswantiningsih IN, Aizawa M, Asanuma K. MAGI-2 and Scaffold Proteins in Glomerulopathy. Am J Physiol Physiol. 2018; 314(2): 181-189. [PubMed] 